\section{Kurz notiert}

Mit Mikronährstoffen gegen Glaskörpertrübung $>$ Im Rahmen einer Anwendungsbeobachtung von Dr. Thomas Kaercher, Augenarzt in Heidelberg, von Juni 2012 bis Januar 2013 erhielten 24 Patienten (im Mittel 62,0 Jahre) über drei Monate täglich eine Kapsel Vitro$\mathrm{Cap}^{\circledR}$, eine Kombination aus Mikronährstoffen, die speziell auf die Bedürfnisse von Patienten mit Glaskörpertrübungen abgestimmt ist.

Mittels einer Spaltlampenuntersuchung wurden die Verklumpungen der Kollagenfasern im Glaskörper als Ursache der Beschwerden verifiziert. Die subjektiven Symptome wurden in fünf Scorestufen erfasst. Der mittlere Anfangsscore lag bei 3,63.

Am Ende der Studie brachten es die Patienten jetzt im Mittel nur noch auf einen Score von 1,33. Zwei Patientinnen bedauerten, dass es keine Null auf der Skala gab, da sie gar keine Beschwerden mehr hatten. $12,5 \%$ empfanden keine Besserung. 87,5\% des Studienkollektivs gaben jedoch eine Besserung der Beschwerden nach dreimonatiger VitroCap ${ }^{\oplus}$-Einnahme an. Dies ist ein möglicher Hinweis, dass eine langfristige diätetische Therapie zur völligen Beschwerdefreiheit bei Mouches Volantes führen kann.

Red. .

- Nach Informationen von ebiga-VISIO

Neue ISO-Norm für Blutzuckermessgeräte - Seit dem 14. Mai 2013 definiert die neue ISO-Norm 15197:2013 strengere Qualitätsstandard bei der Systemgenauigkeit von Blutzuckermesssystemen. Zudem muss die Messgenauigkeit erstmals auch bei der Anwendung des Blutzuckermessgeräts durch Patienten gewährleistet und durch Studien belegt sein. Die einfache Verständlichkeit und Handhabung der Geräte stehen dabei im Fokus. Die Blutzuckermessgeräte der Contour ${ }^{\circledR}$ NEXT Generation erfüllen bereits seit der Markteinführung 2012 die strengeren Anforderungen dieser neuen ISO-Norm.

Red. -

- Nach Informationen von Bayer HealthCare

Diagnostik von Rückenschmerzen

\title{
Ein Fragebogen hilft!
}

- Mit dem Praxis-Fragebogen MyoTect ${ }^{\circledR}$ fällt die Diagnostik bei Patienten mit muskulär bedingten Schmerzen an Schulter, Nacken oder Rücken wesentlich leichter. Entwickelt wurde er von der Deutschen Gesellschaft für Schmerztherapie (DGS). Neben der einseitigen Kurzversion, der ausschließlich Patientenfragen enthält, bietet die vierseitige Version anamnestische Fragen und die Möglichkeit, das Ergebnis der ärztlichen Untersuchung zu dokumentieren.

Trotz seiner mitunter sehr subjektiv erscheinenden Fragen zur sensorischen Schmerzempfindung ist MyoTect ${ }^{\oplus}$ mit einer Sensitivität von $86 \%$ und einem positiven Vorhersagewert von $82 \%$ überaus trennscharf und aufgrund seiner Einfachheit und Kürze vor allem auch extrem praxisfreundlich: In zehn Minuten hat der Patient die leicht verständlichen Fragen beantwortet.
Für jede „Ja“-Antwort des Patienten innerhalb der fünf Fragen gibt es einen Punkt, maximal sind 25 Punkte möglich. Bei mehr als 16 Punkten ist eine muskuläre Ursache des Rückenschmerzes sicher. Damit ist die Indikation gegeben für ein Analgetikum, welches zusätzlich die Muskelverspannung löst. Der MyoTect ${ }^{\oplus}$-Bogen ist so eine wichtige Begründung für die verordnete Therapie gegenüber Dritten.

Eine kostenlose Mappe mit je einem Block kann unter dem Stichwort MyoTect angefordert werden.Per E-Mail: vertrieb@ springer.com oder per Fax: 06102/506-240, Stichwort: MyoTect.

Ein erläuternder Kurzfilm steht im Web unter: www.springermedizin.de/myotect.

Dr. Michael Hubert .

- Quelle: Umfrage von Springer Medizin mit Unterstützung durch TEVA

\section{Unterstützende Behandlung chronischer Wunden}

\section{Die Oxigenierung verbessern}

- Chronische Wunden haben einen hohen Sauerstoffbedarf. Dieser kann jedoch durch äußerliche Applikation nicht gedeckt werden, weil Sauerstoff die Diffusionsbarriere des Wundexsudats nicht überwinden kann. Ein neues Behandlungskonzept ist die Anwendung natürlichen Hämoglobins in wässriger Lösung, das Sauerstoff aus der Umgebungsluft bindet, leicht durch die Barriere diffundiert und so den benötigten Sauerstoff in die Wunde transportiert.

Das erste verfügbare Produkt ist Granulox ${ }^{\oplus}$ : Das Spray enthält 10\% Hämoglobin in 0,9\%iger Kochsalzlösung, das nach der Wundreinigung auf die Wunde gesprüht wird. Anschließend wird die Wunde gemäß der modernen hydroaktiven Wundbehandlung versorgt. Granulox ${ }^{\circledast}$ ist verschreibungs- und erstattungs- fähig und wird adjuvant zur kausalen Therapie der Primärerkrankung eingesetzt.

\section{Vollständige Heilung in kurzer Zeit}

In der Zulassungsstudie in Mexiko mit $42 \mathrm{~Pa}$ tienten konnte durch die Behandlung mit dem Hämoglobinspray bei $93 \%$ der Patienten

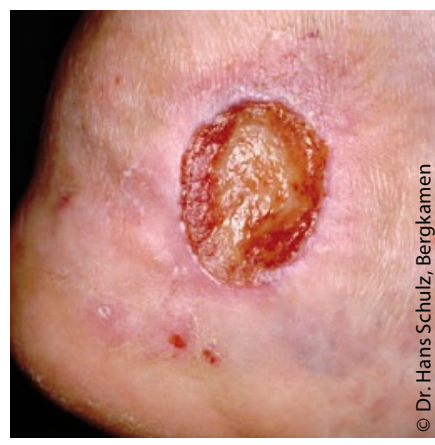

Ulcus cruris venosum. innerhalb von durchschnittlich vier Monaten eine komplette Wundheilung erzielt werden. Die kontrollierte Studie wurde wegen der hohen Effektivität der Behandlung nach sechs Monaten abgebrochen und in eine Beobachtungsstudie umgewandelt, berichtete Dr. Francisco Javier García Luna Martínez, Tamaulipas, Mexiko.

\section{Michael Koczorek}

- Quelle: Wissenschaftliche Vortragsreihe "Granaten, Geld und Granulox - Kosten sparen durch Wirksamkeit"; Deutscher Wundkongress, Bremen, Mai 2013 (Veranstalter: Sastomed) 